\title{
RANDOM SHIFTS WHICH PRESERVE MEASURE ${ }^{1}$
}

\author{
DONALD GEMAN AND JOSEPH HOROWITZ
}

ABSTRACT. Given a flow $\theta_{g}, g \in G$ a group, over a probability space $(\Omega, \mathcal{F}, P)$ and a $G$-valued random variable $Z$, we exhibit the Lebesgue decomposition of the measure $P \circ \theta_{Z}^{-1}$ relative to $P$, and give necessary and sufficient conditions for equality $\left(P \circ \theta_{Z}^{-1}=P\right)$, absolute continuity $\left(P \circ \theta_{Z}^{-1} \ll P\right)$, and singularity $\left(P \circ \theta_{Z}^{-1} \perp P\right.$ ) in terms of the Haar measure. The proof rests on the theory of "Palm measures" as developed by Mecke and the authors. Specializing the group $G$, we retrieve some known results for the integers and real line, and compute the Radon-Nikodým derivatives in various cases.

0 . Introduction. Let $G$ be a locally compact, second countable, Abelian group with Borel $\sigma$-field $\varrho$ and Haar measure $\mu(d g$ ) (or just $d g$ ). A flow $\theta=\left(\theta_{g}\right), g \in G$, on a probability space $(\Omega, \mathcal{F}, P)$ is a group of bimeasurable, measure-preserving bijections $\theta_{g}: \Omega \rightarrow \Omega$ such that $\theta_{0}=$ identity and the mapping $(g, \omega) \rightarrow \theta_{g}(\omega)$ is measurable. If $Z: \Omega \rightarrow G$ is measurable, we write $\theta_{Z}$ for the measurable function $\omega \rightarrow \theta_{Z(\omega)}(\omega), \psi_{Z}(g, \omega)=g+Z$ 。 $\theta_{g}(\omega)$, and $P_{Z}=P \circ \theta_{Z}^{-1}$.

A natural question in the study of flows is to characterize those random variables $Z: \Omega \rightarrow G$ for which $\theta_{Z}$ is a measure-preserving transformation, i.e. $P_{Z}=P$. Neveu [8] gave the solution for the case $G=\mathbf{Z}$ (the integers), and Dinges [1] obtained some special results for $G=\mathbf{R}$ (the real line). Finally, it can be deduced from [6, Satz 4.3] that a sufficient condition for $P_{Z}=P$ is that $\psi_{Z}(\cdot, \omega)$ be a Haar-measure preserving transformation on $G$ for almost every $\omega \in \Omega$.

Here we exhibit the Lebesgue decomposition of $P_{Z}$ relative to $P$ using the theory of "Palm measures" [4], [5]. We can then show that the above condition is also necessary for $P_{Z}=P$, and give necessary and sufficient conditions for $P_{Z}$ to be absolutely continuous relative to $P$ (written $P_{Z} \ll$ $P$ ) or equivalent to $P$ (i.e. have the same sets of measure zero). Finally,

Received by the editors February 19, 1974. $28 \mathrm{~A} 70$.

AMS (MOS) subject classifications (1970). Primary 28A65, 60G10; Secondary

1 This work was partially supported by NSF Grant GP 34485 A 1. 
by making appropriate choices for the group $G$, we retrieve and expand some of the results of Neveu and Dinges mentioned above.

$\S 1$ contains some preliminary work on $\mathrm{Palm}$ measures, the main result is given in $\$ 2$, and $\$ 3$ consists of examples.

If $\mathcal{F}$ is a $\sigma$-field, we shall use the (ambiguous) notation $\xi \in(\mathfrak{F})$ to mean that the function $\xi$ is measurable relative to $\mathcal{F}$, the range being clear from context; $\xi \in(\mathcal{F})_{+}$indicates the range is $[0, \infty)$.

1. Palm measures. A homogeneous random measure (HRM) $\alpha(B, \omega)$ is

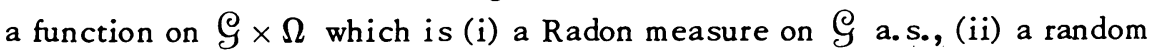
variable on $\Omega$ for all $B \in \mathcal{G}$, and (iii) homogeneous relative to $\theta$ in that $\alpha(B+g, \omega)=\alpha\left(B, \theta_{g} \omega\right) \forall g \in G, B \in \mathcal{Y}$ a.s., where $B+g$ is the translate of $B$ by $g$. (When $G=\mathbf{R}, \alpha((0, t], \omega)$ defines an additive functional.)

Note. Let $N$ and $M$ denote the exceptional $\omega$-sets in (i) and (iii) respectively. Clearly $M$ is $\theta$-invariant; in fact, so is $N \cup M$, so that we can and do assume (i) and (iii) hold off a $P$-null invariant set.

The Palm measure of $\alpha$ is defined by

$$
\hat{P}_{\alpha}(A)=E \int_{G} \zeta(g) I_{A} \circ \theta_{g}(\omega) \alpha(d g, \omega), \quad A \in \mathcal{F},
$$

where $\zeta: G \rightarrow \mathbf{R}$ is such that $\int_{G} \zeta(g) d g=1 . I_{A}$ is the indicator of the set $A$. The measure $\hat{P}_{a}$ is independent of the choice of $\zeta$ (cf. the argument in [5]), and is always $\sigma$-finite. Further, $\hat{P}_{\alpha}$ is finite iff $E \alpha(B)<\infty$ for $B$ compact, in which case $E \alpha(d g)=$ const $\times \mu(d g) ; \alpha$ is then called integrable. A monotone class argument gives

$$
\hat{E}_{\alpha}(\xi)=E \int_{G} \zeta(g) \xi \circ \theta_{g}(\omega) \alpha(d g, \omega), \quad \xi \in(\mathcal{F})_{+},
$$

$\hat{E}_{a}$ being integration with $\hat{P}_{\alpha}$.

Proceeding exactly as in the case $G=\mathbf{R}[4]$ one obtain s

$$
E \int_{G} Y\left(g, \theta_{g} \omega\right) \alpha(d g, \omega)=\hat{E}_{\alpha} \int_{G} Y(g, \omega) d g, \quad Y \in(\varsubsetneqq \otimes \mathcal{F})_{+} \cdot
$$

Choosing $Y(g, \omega)=I_{B}(g) \xi \circ \theta_{-g}(\omega)$, we have $E(\xi \propto(B))=\hat{E}_{a} \int_{B} \xi_{\circ} \circ \theta_{-g} d g$, $B \in \mathcal{G}, \xi \in(\mathfrak{F})_{+}$. Since $\mathcal{G}$ is separable, this leads to

(3) Lemma. Let $\alpha, \beta$ be HRM's. Then $\hat{P}_{\alpha}=\hat{P}_{\beta}$ iff $\alpha(d g, \omega)=\beta(d g, \omega)$ for almost every $\omega \in \Omega$.

From (1) we get

(4) Lemma. Let $Q$ be a $\sigma \cdot f$ inite measure on $\mathcal{F}$ such that $Q \ll \hat{P}_{a}$ for 
some HRM $a$. Then $Q=\hat{P}_{\beta}$, where $\beta(d g, \omega)=\xi \circ \theta_{g}(\omega) \alpha(d g, \omega)$ and $\xi=$ $d Q / d \hat{P}_{\alpha}$.

Before going on, let us observe that any $\operatorname{HRM} \alpha(d g, \omega)$ is $\sigma$-finite on S a.s., hence, using [7, p. 154], it is not difficult to prove that there exists a function $\chi \in(乌 \otimes F)+$ which, for such $\omega$, serves as the density of the $\mu$ absolutely continuous part of $\alpha: \alpha(d g, \omega)=\chi(g, \omega) d g+\gamma(d g, \omega)$, where $\gamma(d g, \omega)$ is a.s. $\mu$-singular (written $\gamma(d g, \omega) \perp \mu(d g)$ a.s.).

(5) Theorem. Let $\beta(d g, \omega)=\chi(g, \omega) d g$ be the $\mu$-absolutely continuous part of $\alpha$, and $\gamma(d g, \omega)$ the $\mu$-singular part. Then $\beta$ and $\gamma$ are HRM's and $\hat{P}_{a}=\hat{P}_{\beta}+\hat{P}_{\gamma}$ is Lebesgue decomposition of $\hat{P}_{a}$ relative to $P: \hat{P}_{\beta} \ll P$, $\hat{P}_{\gamma} \perp P$.

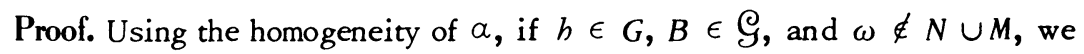
have

$$
\begin{aligned}
\alpha(B+b, \omega) & =\int_{B+h} \chi(g, \omega) d g+\gamma(B+b, \omega) \\
& =\int_{B} \chi(g+b, \omega) d g+\gamma(B+b, \omega) \\
& =\int_{B} \chi\left(g, \theta_{h} \omega\right) d g+\gamma\left(B, \theta_{h} \omega\right) .
\end{aligned}
$$

Now $\gamma(B+h, \omega)$ is $\mu$-singular (as a measure in $B$ ), so, by uniqueness of Lebesgue decomposition, $\gamma(B+h, \omega)=\gamma\left(B, \theta_{h} \omega\right)$, i.e. $\gamma$ is an HRM. Hence $\beta$ is also an HRM, and, obviously, $\hat{P}_{a}=\hat{P}_{\beta}+\hat{P}_{\gamma}$.

Let $\zeta: G \rightarrow \mathbf{R}$ have $\int_{G} \zeta(g) d g=1$. For any $\xi \in(\mathcal{F})_{+}$, we then have

$$
\hat{E}_{\beta}(\xi)=E \int_{G} \zeta(g) \chi(g, \omega) \xi \circ \theta_{g}(\omega) d g=E \xi \int_{G} \zeta(g) \chi\left(g, \theta_{-g} \omega\right) d g
$$

which shows $\hat{P}_{\beta} \ll P$. Since $\zeta$ is arbitrary we note al so that $\chi\left(g, \theta_{-g}(\omega)\right)$ is a.s. constant in $g$, so we can take $\hat{E}_{\beta}(\xi)=E(\xi \chi), \chi \in(\mathcal{F})_{+}$, and, by (3), $\beta(d g, \omega)=\chi \circ \theta_{g}(\omega) d g$ a.s.

Suppose, next, that $\hat{P}_{\gamma}$ is not $P$-singular. Write the Lebesgue decomposition $\hat{P}_{\gamma}=Q+Q^{\prime}$, where $Q \ll P$ and $Q^{\prime} \perp P$. By (4), both $Q$ and $Q^{\prime}$ are $\mathrm{Palm}$ measures. Since $P$ is the $\mathrm{Palm}$ measure of $\mu(d g)$, (4) tells us that $Q$ corresponds to a (nontrivial) HRM of the form $\eta \circ \theta_{g} d g$. Let $\rho$ be the HRM corresponding to $Q^{\prime}$. Then, by (3), $\gamma(d g, \omega)=\eta \circ \theta_{g}(\omega) d g+\rho(d g, \omega)$ a.s., contradicting the a.s. $\mu$-singularity of $\gamma$. Q.E.D.

(6) Corollary. For an HRM a, $\hat{P}_{\alpha} \ll P$ (resp. $\hat{P}_{\alpha} \perp P$ ) iff $\alpha(d g, \omega) \ll$ $\mu(d g)$ a.s. (resp. $a(d g, \omega) \perp \mu(d g)$ a.s. $)$. 
2. Main result. Let $Z, \psi_{Z}$, and $P_{Z}$ be as in $\S 0$, and define a random measure by

$$
\alpha_{Z}(B, \omega)=\int_{G} I_{B}\left(\psi_{Z}(g, \omega)\right) d g, \quad B \in \oint .
$$

(8) Lemma. The random measure $\alpha_{Z}$ is an HRM whose Palm measure is $P_{Z}$.

Proof. First consider

$$
\begin{aligned}
E \alpha_{Z}(B) & =E \int_{G} I_{B}\left(g+Z \circ \theta_{g}\right) d g \\
& \left.=E \int_{G} I_{B}(g+Z) d g \quad \text { (since } \theta_{g} \text { is a flow }\right) \\
& =\mu(B) .
\end{aligned}
$$

Using the $\sigma$-compactness of $G$, we find that $\alpha_{Z}(B, \omega)$ is finite on compacts a.s. Next, to prove homogen eity, fix $h \in G$. We have

$$
\begin{aligned}
a_{Z}(B+h, \omega) & =\int_{G} I_{B+h}\left(g+Z \circ \theta_{g}(\omega)\right) d g=\int_{G} I_{B}\left(g-b+Z \circ \theta_{g}(\omega)\right) d g \\
& =\int_{G} I_{B}\left(g+Z \circ \theta_{g+h}(\omega)\right) d g \quad\left(\text { since } \theta_{g+h}=\theta_{g} \circ \theta_{h}\right) \\
& =a_{Z}\left(B, \theta_{h} \omega\right) .
\end{aligned}
$$

Finally, it is clear from (7) that, for any function $\phi \in\left(\bigodot_{+}\right.$,

$$
\int_{G} \phi(g) \alpha_{Z}(d g, \omega)=\int_{G} \phi\left(\psi_{Z}(g, \omega)\right) d g .
$$

It follows that, if $\xi \in(\mathfrak{F})_{+}$and $\zeta: G \rightarrow \mathbf{R}$ satisfies $\int_{G} \zeta(g) d g=1$,

$$
\begin{aligned}
& \hat{E}_{a_{Z}}(\xi)=E \int_{G} \zeta\left(g+Z \circ \theta_{g}\right) \xi \circ \theta_{g+Z \circ \theta_{g}} d g \\
&=E \int_{G} \zeta\left(g+Z \circ \theta_{g}\right) \xi \circ \theta_{Z} \circ \theta_{g} d g \\
&=E \int_{G} \zeta(g+Z) \xi \circ \theta_{Z} d g=\int \xi d P \\
& Z
\end{aligned}
$$

Note. Since $a_{Z}(d g, \omega)$ is homogen eous $\forall \omega$ (i.e. $\left.M=\varnothing\right)$ and a measure $\forall \omega$, and since $G$ has a countable basis of relatively compact sets, we need not complete $\mathcal{F}$ to ensure the measurability of the $\omega$-set on which $a_{Z}(d g, \omega)$ is finite on compacts.

As a consequence of (8), if $\beta(d g, \omega)+\gamma(d g, \omega)$ is the Lebesgue decomposition of $\alpha_{z}(d g, \omega)$ relative to $\mu(d g)$, where $\beta(d g, \omega) \ll \mu(d g)$ and $\gamma(d g, \omega) \perp \mu(d g)$ a.s., we find the Lebesgue decomposition 


$$
P_{Z}=\hat{P}_{\beta}+\hat{P}_{\gamma}, \quad \hat{P}_{\beta} \ll P, \quad \hat{P}_{\gamma} \perp P .
$$

Explicit expressions for $\hat{P}_{\beta}$ will be given in some special cases in $\$ 3$.

Finally, using the results of $\S 1$, namely (3), (4), (6), and noting that $a_{Z}(d g, \omega)$ is the measure $\mu \circ \psi_{Z}^{-1}(\cdot, \omega)$, we have

(10) Theorem. (a) $P_{Z}=P$ iff $\mu \circ \psi_{Z}^{-1}(\cdot, \omega)=\mu$ a.s., i.e. iff the function $\psi_{Z}(\cdot, \omega)$ is $\mu-m e a s u r e$ preserving a.s.

(b) $P_{Z} \ll P$ iff $\mu \circ \psi_{Z}^{-1}(\cdot, \omega) \ll \mu$ a.s.

(c) $P_{Z}$ is equivalent to $P$ iff $\mu \circ \psi_{Z}^{-1}(\cdot, \omega)$ is equivalent to $\mu$ a.s.

3. Examples. 1. Let $G$ be any countable group with the discrete topology, $\mu$ the counting measure. If $\alpha$ is any $\mathrm{HRM}$ and $\xi \in(\mathcal{F})_{+}$, we have from (1):

$$
\begin{aligned}
\hat{E}_{\alpha}(\xi) & =E \sum_{k \in G} \zeta(k) \xi \circ \theta_{k} \alpha(\{k\}) \\
& =\sum_{k \in G} \zeta(k) E\left(\xi(\omega) \alpha\left(\{k\}, \theta_{-k} \omega\right)\right)=E(\xi \alpha(\{0\}))
\end{aligned}
$$

where $\Sigma \zeta(k)=1$. Hence $\hat{P}_{a} \ll P$. In particular, $P_{Z} \ll P$ and the RadonNikodým derivative is

$$
\alpha_{Z}(\{0\})=\sum_{k \in G} I_{\{0\}}\left(k+Z \circ \theta_{k}\right)=\#\left\{k \in G: \psi_{Z}(k)=0\right\},
$$

where \# denotes the cardinality $(\leq+\infty)$ of the indicated set. For $P_{Z}=P$ it is then necessary and sufficient to have $1=\Sigma_{k \in G} I_{\left\{k+Z_{0} \theta_{k}=0\right\}}$ a.s., a result due to Neveu [8] when $G=Z$.

2. Let $G$ be arbitrary, but suppose that $Z$ is discrete. Computing $P_{Z}$ directly, it happens again that $P_{Z} \ll P$ with derivative $\eta(\omega)=$ $\#\left\{g \in G: \psi_{Z}(g, \omega)=0\right\}$.

3. Consider the case $G=\mathbf{R}$ and assume $Z(\omega) \geq 0$ is a "terminal" random variable, meaning that $Z(\omega)=t+Z \circ \theta_{t}(\omega)$ whenever $Z(\omega)>t, t \in \mathbf{R}$. Consequently, $\alpha_{Z}(\{Z(\omega)\}, \omega) \geq Z(\omega)$. Thus, $\alpha(d t, \omega)$ has a singular component whenever $Z(\omega)>0$, so that $P_{Z} \ll P$ is impossible unless $Z=0$ a.s., in which case $P_{Z}=P$.

If $M(\omega)$ is a homogeneous random set, i.e. $M\left(\theta_{t} \omega\right)=M(\omega)-t$ for all $t$, $\omega$, then $Z(\omega)=\inf \{t>0: t \in M(\omega)\}$ is terminal, and an easy computation yields, for any $t>0$,

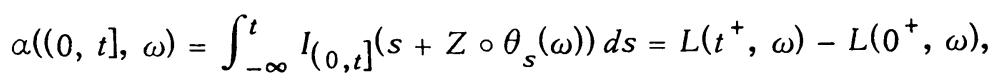


where $L(t, \omega)=\sup \{s \leq t: s \in M(\omega)\}$. By (8), the Palm measure of the additive functional $L\left(t^{+}\right)-L\left(0^{+}\right)$is just $P \circ \theta_{Z}^{-1}$.

4. Consider again $G=\mathbf{R}$, and suppose now that, for almost every $\omega \in \Omega$, $Z \circ \theta_{t}(\omega)$ is an absolutely continuous function of $t \in \mathbf{R}$. By a Fubini argument it is easy to prove that there is a random variable $Z^{\prime}$ such that $Z^{\prime}$ 。 $\theta_{t}(\omega)=d Z \circ \theta_{t}(\omega) / d t$ for almost every $t$, a.s. The process $\psi_{Z}(t, \omega)=t+$ $Z \circ \theta_{t}(\omega)$ is absolutely continuous and a.s. has derivative $\psi^{\prime}(t, \omega)=1+$ $Z^{\prime} \circ \theta_{t}(\omega)$ a.e. To say that $a_{Z}(\cdot, \omega) \ll \mu$ means that, if $\mu(B)=0$ for $B \epsilon$ $\mathcal{B}$ (the Borel $\sigma$-field on $\mathbf{R}$ ), then the process $\psi(t, \omega)=\psi_{Z}(t, \omega)$ spends (Lebesgue measure) zero time in $B$, i.e. $\psi(t, \omega)$ has a local time relative to $\mu$. Local times (or occupation time densities) for smooth processes were studied in [3], and we now apply some of those results to the process at hand. Let

$$
\begin{gathered}
\nu_{x}(U, \omega)=\#\{s \in U: \psi(s, \omega)=x\}, \quad U \in \mathbb{B}, \\
\beta(x, \omega)=\int_{-\infty}^{\infty}\left|\psi^{\prime}(s, \omega)\right|^{-1} \nu_{x}(d s, \omega) .
\end{gathered}
$$

Now $a_{Z}(B, \omega)=\int_{-\infty}^{\infty} I_{B}(\psi(t, \omega)) d t$ is the total time spent by $\psi(\cdot, \omega)$ in $B$. From [3] we conclude that $\beta(x, \omega)$ is the density of the absolutely continuous component of the measure $\alpha_{z}(\cdot, \omega)$. A necessary and sufficient condition for the absence of the singular component is that $\mu\left\{t: \psi^{\prime}(t, \omega)=0\right\}=0$ a.s. By a Fubini argument and stationarity, this reduces to $P\left\{Z^{\prime}=-1\right\}=0$.

Consider the absolutely continuous component $\alpha_{a}$ of $\alpha_{Z}$ in more detail. We have

$$
\begin{aligned}
\beta(x, \omega) & =\int_{-\infty}^{\infty}\left|1+Z^{\prime} \circ \theta_{s}\right|^{-1} \nu_{x}(d s, \omega)=\sum_{s: s+Z \circ \theta_{s}=x}\left|1+Z^{\prime} \circ \theta_{s}\right|^{-1} \\
& =\sum_{s: \psi\left(s, \theta_{x} \omega\right)=0}\left|1+Z^{\prime} \circ \theta_{s} \circ \theta_{x}(\omega)\right|^{-1}=\beta\left(0, \theta_{x} \omega\right) .
\end{aligned}
$$

Thus $\alpha_{a}(d t, \omega)=\beta\left(0, \theta_{t} \omega\right) d t$, so the Palm measure of $\alpha_{a}$ is $\hat{P}_{a_{a}}(A)=$ $E(\beta(0) ; A), A \in \mathcal{F}$, i.e. the density of the absolutely continuous component of $P_{Z}$ is $\beta(0)=\int_{-\infty}^{\infty}\left|1+Z^{\prime} \circ \theta_{s}\right|^{-1} \nu_{0}(d s)$. This appears to generalize some of the work of Dinges [1], though we have been unable to comprehend fully his results. It is also a continuous analogue of Example 2.

Finally we note that, if $Z$ is integrable, $\beta(0)>0$ a.s. and so $\alpha_{a}$ is a.s. equivalent to $\mu$, i.e. $\hat{P}_{a}$ is equivalent to $P$. Indeed, it may be shown that, a.s., the derivative $\psi^{\prime}(s, \omega)$ is finite at each time $s$ at which $\psi(s, \omega)=x$, 
for a.e. $x$. It remains only to show that $\psi(\cdot, \omega)$ hits every $x$, a.s. Suppose $\psi(s, \omega)<x$ for every $s$. Then $t^{-1} \cdot \int_{0}^{t} \psi(s, \omega) d s<x$ for all $t$. But $t^{-1} \int_{0}^{t} \psi(s, \omega) d s=t / 2+t^{-1} \int_{0}^{t} Z \circ \theta_{s}(\omega) d s$, and this converges, for almost every $\omega$, to $\pm_{\infty}$ as $t \rightarrow \pm_{\infty}$.

5. We indicate here an extension of the result of Example 4 when $G=$ $\mathbf{R}^{n}, n \geq 1$. Assume that $t \rightarrow Z \circ \theta_{t}(\omega)$ is a.s. a Lipschitz function of $t \epsilon$ $\mathbf{R}^{n}$. Then $\psi(t, \omega)=t+Z \circ \theta_{t}(\omega)$ is likewise, and Theorem 3.2.5 (see also 2.10.35) of [2] yields (omitting $\omega$ )

$$
\int_{U} g(\psi(t)) J(t) d t=\int_{\mathbf{R}^{n}} g(y)_{\nu_{y}}(U) d y \text { a.s. }
$$

for any Borel function $g$ (Borel set $U$ ) on $\mathbf{R}^{n}$, where $J(t)$ is the absolute value of the Jacobian of $\psi(t)$, which exists a.e., and $\nu_{y}(U)$ is defined as in Example 4. Arguing as in $\$ 1$ of [3] we find

$$
\int_{\mathbf{R}^{n}} b(t) g(\psi(t)) J(t) d t=\int_{\mathbf{R}^{n}} g(y) \int_{\mathbf{R}^{n}} b(t) \nu_{y}(d t) d y
$$

for any nonnegative Borel function $h(t)$. Let $N$ be a Borel set which is almost equal to $\{t: J(t)=0\}$ (which is, in general, only Lebesgue measurable). Then [2, Theorem 3.2.3] shows $\nu_{y}(N)=0$ for a.e. $y$. Now take $h(t)=$ $(J(t))^{-1}{ }_{N} c^{(t)}$ a.e. From (12) we have

$$
\int_{\mathbf{R}^{n}} g(\psi(t)) I_{N^{c}}(t) d t=\int_{\mathbf{R}^{n}} g(y) \int_{\mathbf{R}^{n}} \frac{\nu_{y}(d t)}{J(t)} d y,
$$

or, with $g=I_{B}$,

$$
\int_{N^{c}} I_{B}(\psi(t)) d t=\int_{B} \int_{\mathbf{R}^{n}} \frac{\nu_{y}(d t)}{J(t)} d y .
$$

As in [3], one notes that the left member of (13) is the absolutely continuous component of $a_{Z}$, and we conclude: the $P$-absolutely continuous component of $P_{Z}$ has density $\int_{\mathbf{R}^{n}} \nu_{0}(d t) / J(t)$.

\section{REFERENCES}

1. H. Dinges, Random shifts of stationary processes, Proc. Fifth Berkeley Sympos. Math. Statist. and Probability (Berkeley, Calif., 1965/66), vol. 2: Contributions to Probability Theory, part 1, Univ. of California Press, Berkeley, Calif., 1967, pp. 99-116. MR 35 \#2359.

2. H. Federer, Geometric measure theory, Die Grundlehren der math. Wissenschaften, Band 153, Springer-Verlag, New York, 1969. MR 41 \#1976. 
3. D. Geman and J. Horowitz, Occupation times for smooth stationary processes, Ann. of Prob. 1 (1973), 131-137.

4. - Remarks on Palm measures, Ann. Inst. H. Poincaré 9 (1973), 215232.

5. J. Mecke, Stationäre zufällige Masse auf lokalkompakten Abelschen Gruppen, Z. Wahrscheinlichkeitstheorie und Verw. Gebiete 9 (1967), 36-58. MR 37 \#3611.

6. - Invarianzeigenschaften allgemeiner Palmsche Masse (to appear).

7. P. A. Meyer, Probability and potentials, Blaisdell, Waltham, Mass., 1966. MR $34 \# 5119$.

8. J. Neveu, Temps d'arret d'un système dynamique, Z. Wahrscheinlichkeitstheorie und Verw. Gebiete 13 (1969), 81-94. MR $41 \# 427$.

DEPARTMENT OF MATHEMATICS, UNIVERSITY OF MASSACHUSETTS, AMHERST, MASSACHUSETTS 01002 